This item was submitted to Loughborough's Research Repository by the author.

Items in Figshare are protected by copyright, with all rights reserved, unless otherwise indicated.

\title{
A survey of expert opinion on the effects of occupational exposures to trunk rotation and whole-body vibration
}

PLEASE CITE THE PUBLISHED VERSION

http://dx.doi.org/10.1080/00140139.2014.887785

PUBLISHER

(c) Taylor \& Francis

VERSION

AM (Accepted Manuscript)

LICENCE

CC BY-NC-ND 4.0

\section{REPOSITORY RECORD}

Morgan, Lauren J., and Neil J. Mansfield. 2019. "A Survey of Expert Opinion on the Effects of Occupational Exposures to Trunk Rotation and Whole-body Vibration". figshare. https://hdl.handle.net/2134/14498. 
This item was submitted to Loughborough's Institutional Repository (https://dspace.lboro.ac.uk/) by the author and is made available under the following Creative Commons Licence conditions.

\section{creative
commons}

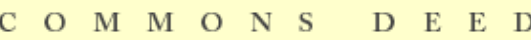

Attribution-NonCommercial-NoDerivs 2.5

You are free:

- to copy, distribute, display, and perform the work

Under the following conditions:

Attribution. You must attribute the work in the manner specified b the author or licensor.

Noncommercial. You may not use this work for commercial purposes.

No Derivative Works. You may not alter, transform, or build upon this work.

- For any reuse or distribution, you must make clear to others the license terms of this work.

- Any of these conditions can be waived if you get permission from the copyright holder.

Your fair use and other rights are in no way affected by the above.

This is a human-readable summary of the Leqal Code (the full license).

\section{Disclaimer 만}

For the full text of this licence, please go to: http://creativecommons.org/licenses/by-nc-nd/2.5/ 


\section{Ergonomics}

Volume 57, Issue 4, 2014 pages 563-574

DOI:10.1080/00140139.2014.887785

A survey of expert opinion on the effects of occupational exposures to trunk rotation and whole-body vibration

Lauren J Morgan and Neil J Mansfield*

Loughborough Design School

Loughborough University

Loughborough

UK

LE11 3TU

01509226925

* Corresponding author - lauren.morgan@nds.ox.ac.uk 


\title{
A survey of expert opinion on the effects of occupational exposures to trunk rotation and whole-body vibration
}

\begin{abstract}
We present a review of current expert opinion on the effects of combined exposures to trunk rotation and whole-body vibration, commonly experienced by operators of agricultural machinery. We evaluate the level of agreement between academic experts in the field of ergonomics, human response to whole-body vibration, and agricultural operators, on the effects of exposure to whole-body vibration (WBV) and trunk rotation. A total of 83 individuals responded to the paper-based questionnaire, that included questions on risk levels from individual and combined exposures, discomfort development, exposure duration limits and tasks within agriculture. The results showed that all groups considered exposure to WBV and trunk rotation as risk factors for the development of back pain. The experts were not in consensus regarding acceptable exposure durations, areas of discomfort experienced or recommendations for cab developments.
\end{abstract}

Key Words: Agriculture; vibration; driving; trunk rotation; low back pain

Practitioner summary: Trunk rotation combined with whole-body vibration are suggested causes for low back pain in exposed populations. The expert opinion on any possible interactions is explored through risk assessment models and recommendations for future practice. Designers should consider paying greater attention to operator's feedback on the machines they use, as this may include valuable insight to how to improve future developments. 


\section{Introduction}

Low back pain (LBP) is a major cause of morbidity across high-, middle- and low income countries (Hoy et al., 2010), yet there is little information concerning the global burden of non-traumatic LBP attributable to the effects of physical occupational exposures (Punnett et al., 2005). To date the issue of low back pain in agriculture is considered relatively under prioritised and under-funded in the fields of occupational health (Hoy et al., 2010). Poor and/or long term seated postures are known to contribute to the development of symptoms in the low back (Pheasant and Haslegrave, 2006). In particular, trunk rotation (twist) has been found to be significantly related to low back pain (Frymoyer, et al., 1998). In addition to the posture of the driver, there may be other factors in a work system that cause stress upon the body. In agriculture, one of these additional stressors is whole-body vibration (WBV) transmitted to the worker, which is widely accepted as a risk factor for LBP amongst exposed populations (Wikstrom, et al. 1994; Lings \& Leboeuf-Yde, 2000). Increased lifetime vibration dose increases the risk of developing a low back condition (Schwarze, et al. 1998; Tiemessen, et al. 2008; Bovenzi, 2009), with sufferers often experiencing not only discomfort but pain and disability.

It has been suggested that combinations of vibration and postural stress increase the likelihood of the development of low back pain (Donati, 2002). The posture of the operator may make the muscles and circulatory system less tolerant to the stresses of vibration (Magnusson and Pope, 1998). The lumbar vertebral discs exhibit increased fracture risk under both vibration and constrained postural stress (Wilder and Pope, 1996). Some researchers are beginning to support the concept that it is the interaction effects of posture, vibration and/or manual materials handling that are the main contributors for precipitation of LBP (Okunribido, et al., 2008). The exposure-response relationship between combined trunk 
rotation, WBV and associated health risk has proven difficult to identify. ISO 2631-1 (1997) requires that operator posture is taken into consideration but provides no criteria by which judgments can be made.

Within industrialised agriculture, few tasks are carried out without agricultural wheeled tractors. These have rear mounted linkages and power take-off where machinery, implements and trailers are attached; controls for these are banked to the right of the operator within the cab. Depending on the task, operators may be required to visually monitor rear-mounted implements, operate in-cab controls, and maintain vehicle control. This combination of factors dictates the operating posture, often resulting in prolonged periods of trunk rotation (Bottoms and Barber, 1978; Mehta and Tiwari, 2000). Vibration exposures within agriculture are also known to exceed the guidance for acceptable daily exposures (Sorainen et al., 1998). The three-year averaged prevalence for LBP in agriculture is reported as 1410 per 100,000 employed (HSE, 2007/8), which is the largest prevalence of all industries in the UK.

The fields of whole-body vibration and postural ergonomics research are well founded, and an extensive literature is present for each area. However, the cross-over area covering occupations with both risk factors is less well developed. It is suggested that many experts working within these areas may have experience and opinions that are often not contained within the academic literature, and therefore cannot be elicited from a standard literature review. The skills used by an expert to formulate an opinion develop over many years of concentrated training and research and as such their thoughts and views on a topic are central to understanding the issue. Therefore, to advance the understanding in the broader topic area, a survey of expert opinion covering experts from both areas would allow a rapid review of available knowledge. Additionally, considering the knowledge and experience of the 
operators themselves, will add a unique perspective.

The method of surveying expert opinion on issues in ergonomics and in agriculture is not common, although it was used by Sandover (1998). Sandover considered three linked investigations to increase understanding of high acceleration and shock events. His approach included a literature review, field data, and a review of expert opinion. In addition, others have used expert opinion of risks as a basis for the development of many posture assessment tools (Hignett and McAtamney, 2000, David et al., 2008). This study forms part of a wider research project on understanding the risks from combined exposure to WBV and trunk rotation. The purpose of this study was to collate unpublished knowledge and opinions of experts on the presence and magnitude of the risks surrounding simultaneous exposure to trunk rotation and WBV. A secondary objective was to determine where conflictions in opinion/judgement may occur between the expert groups.

\section{Methods}

The three expert groups chosen for analysis were: those working within the area of evaluation and assessment of WBV, vibration experts (VE); ergonomists, particularly with postural ergonomics experience, postural experts (PE); and operators with experience of exposures consisting of trunk rotation and WBV, operator experts (OE). A questionnaire was developed so that most questions could be answered by the three respondent groups; however it was appropriate to remove/supplement some questions in the operator (OE) questionnaire. The questionnaire was formulated for completion either online (Survey Monkey, 2009) or via a paper based form. This combination of collection methods increases the range of possible respondents and maximises the response rate (Gosling et al., 2004). Various methods of recruitment were utilised to ensure a fair representation of the target population. These are 
shown in Table 3 along with the principle method of completion for that cohort.

\section{[TABLE 1 ABOUT HERE]}

No monetary incentives were provided to participants for completion of the survey.

The survey introduction included a summary of the topic area, thanked participants and contained Loughborough University logo and contact details to reinforce the source of the questionnaire. Participants were also notified of the presence of comment boxes throughout the survey and encouraged to write any additional information in these that they felt appropriate. The confidentiality and withdrawal information was repeated along with researcher contact details. Ethical approval for the study was granted locally by Loughborough University ethical advisory committee. To facilitate online completion, a recruitment e-mail and posts on discussion forums asked professionals to participate in a survey of occupational exposures in agriculture.

The survey itself was organised into five sections, from sections of the literature. Demographics, although not essential to the problem understanding, were included to assist in the interpretation of results.

\subsection{Respondent demographics}

A demographics section contained questions on the area and length of expertise of the respondent.

- PE respondents were asked to indicate their main area of expertise (from IEHF, 2008): Anatomy, anthropology and physiology in human activities (incl. postural and biomechanical loading) 
- Environmental stressors (incl. vibration)

- General psychology \& organisational psychology (incl. cognitive ergonomics)

- Socio-technical systems (incl. systems evaluation)

- Survey and research methods (incl. measurement techniques)

OE respondents were asked to indicate their main area of expertise (from DEFRA, 2008):

- Arable

- Contractor

- Dairy

- Livestock

- Poultry

- Pigs

The addition of contractors was made to the DEFRA categories, as although not considered 'farmers' by DEFRA, this operator group have extensive experience of the exposures.

An option for 'other' was also provided in both cases, although respondents choosing this box were asked to specify. Subjects were asked to indicate their length of experience in broad response categories, (none; < 1 year; 1-2 years; 2-5 years; 5-10 years; >10 years).

Operator experts (OE) were asked to estimate their typical driving time (per day) during busy periods. Due to the variable nature of work in agriculture it was considered necessary to specify 'busy periods' to get a true representation of time spent on tractors when using them. They were also asked to estimate the number of rest periods typically taken during this driving activity.

\subsection{Health risks}


It is difficult to compare risks from different exposures if those exposures are not risk assessed in the same way, or to the same criterion. A similar method to the development of postural assessment methods (McAtamney et al., 1993;Hignett and McAtamney, 2000) is employed here. This is to rank exposures to facilitate a comparison between them.

Descriptions of the exposures were provided, rather than illustrations, as vibration magnitude could not be easily conveyed via illustrations and this may have caused unnecessary bias. The categories of exposure were:

- No twist, low vibration (smooth driving $\sim 0.25 \mathrm{~m} / \mathrm{s}^{2}$ )

- No twist, medium vibration (rough driving $\sim 0.5 \mathrm{~m} / \mathrm{s}^{2}$ )

- No twist, high vibration (off road driving $\sim 1 \mathrm{~m} / \mathrm{s}^{2}$ )

- Medium twist $\left(70^{\circ}\right)$, low vibration

- Medium twist, medium vibration

- Medium twist, high vibration

- High twist $\left(170^{\circ}\right)$, low vibration

- High twist, medium vibration

- High twist, high vibration

The experts were asked to consider each of the exposures and to rate how risky they felt each exposure would be for the development of a musculoskeletal disorder, including those of the low back (therefore resulting in LBP). The verbal descriptives for risk level are taken from the verbal anchors in ISO2631-1 (1997). It is a unipolar 5 point scale, ranging from not at all to extremely. The response categories were as detailed below:

- Not at all

- Slightly

- Moderately 
- Very

- Extremely

Only PE and VE were asked to complete this question. All experts were asked to classify whether they thought a risk for low back pain was posed by trunk rotation and by WBV separately. To establish if it was felt an interaction effect existed, they were also asked to consider if they felt the risk increased if the exposures were combined.

\subsection{Risk Assessment}

The use of exposure limitation is a common method of risk control. Guidance and standards often prescribe a limit on exposure duration e.g. Physical Agents (Vibration) Directive (PA(V)D) (European Commission, 2002). In the PA(V)D the exposure duration and exposure magnitude are considered proportional to the perceived risk, whereby a high vibration magnitude short duration exposure could be considered equivalent to a low vibration magnitude long duration exposure. The relative equivalence is calculated using a dose measure, the $A(8)$. A method often employed to determine the severity of exposure is to ask operators to consider "how long would you do this for?" (Wikstrom, 1993). Here, experts were asked to consider what they felt acceptable exposure durations would be for a variety of twist and vibration combinations:

- No twist, no vibration

- Medium twist, no vibration

- High twist, no vibration

- No twist, with vibration

- Medium twist, with vibration

- High twist, with vibration

No response categories were provided to avoid biasing the responses, experts were asked to 
respond in free text.

\subsection{Discomfort development}

Subjective discomfort is used in many studies to rate exposures. More specifically, body part discomfort has been used to investigate patterns of discomfort, in the investigation of comparable exposures (Kuorinka et al., 1987). Experts were asked to mark on a body outline where they felt discomfort would manifest in three separated exposure conditions: wholebody vibration only; whole-body vibration and trunk rotation; trunk rotation only. The body map used is shown in Figure 1.

[FIGURE 1 ABOUT HERE]

Experts were also asked to consider at which stage (guidance, standards or legislation) posture should be included if at all. This question was omitted from the OE questionnaire, as it was deemed unsuitable. Those with experience in assessing WBV were asked whether they considered that the posture of the operator should be reflected as part of the assessment of WBV exposure.

\subsection{Evaluation of previous research}

A series of developments in both the design of the work system, and the vehicles themselves have been suggested by the literature over many decades, these have included swivelling seat bases (Bottoms and Barber, 1978; Toren and Oberg, 2001) and enlarged mirrors (Sjofløt, 1980). The use of full backrest seats are often recommended by those investigating vibration exposures (Control of Vibration at Work Regulations, 2005). To investigate the perceived effectiveness of such recommendations, a common task scenario was put to the experts and they were asked whether they would recommend or not the addition of a swivelling seat base, 
a full backrest seat, enlarged mirrors or CCTV for a driver to complete the task. The common task was selected so that it would deliberately include high duration and frequency of twists. The task description is given below:

- “An agricultural driver is required to plough an uneven field. This task requires constant rear and forward attention. The task will take approximately 8 hours, during which time the driver will leave the cab for 30mins to have lunch.”

A box for comments was also provided.

At the end of the questionnaire, participants were invited to add any further comments about the topic area in general which had not been covered by the questions.

\section{Results}

The response rate varied with the expert groups from $27 \%$ for the vibration experts (13/35 responses) to $70 \%$ for the posture experts (37/53). Due to the nature of recruitment for some of the $\mathrm{OE}$ response group (online posting in discussion forums), it is not possible to determine a definitive response rate, however 74 individuals completed the survey.

\subsection{Demographics}

The length of experience of the response groups was high, with at least $50 \%$ in all response groups having more than 10 years' experience. One OE reported less than 1 years’ experience, but was included in the analysis. This was due to the fact their driving hours per day was high, and they had much experience of the kind of tasks being investigated. It was therefore considered that they could still be considered an 'expert' in these specific exposures. The overview of the experience across the expert groups is illustrated in Figure 2. [FIGURE 2 ABOUT HERE] 
The distribution of expertise amongst the PEs was relatively even, with $>10 \%$ of respondents within each area of expertise. There were a greater proportion of respondents within the 'anatomy, anthropology and physiology' and in the 'other' sections, however this was expected due to the required selection bias towards those with greatest experience in assessing occupational exposures. Of the VE respondents, 67\% had experience in assessing exposures. Within the operator experts, the split amongst the main farming sectors are as follows; arable, 28\%; livestock, 31\%; dairy, $8 \%$; poultry, $4 \%$; not stated (2\%) and contracting, 27\%. The distribution between the main areas of expertise matches the percentage of agricultural land use in the UK as reported by the Department for Environment, Farming and Rural Affairs (DEFRA, 2008). The relatively small contribution of poultry farmers is expected to be due to the low requirement for tractors within poultry farming, and that this sector of farming tends to be in addition to the primary farming method (e.g. arable).

The majority (61\%) of OE reported driving a tractor for in excess of 10 hours a day; in addition $26 \%$ of drivers reported not taking any breaks during the day. The majority took one break per day (28\%), 26\% took two breaks per day, and 14\% took three breaks per day (6\% did not provide this information). No respondent reported taking more than three breaks during the day. Some reported that they continued to work during a 'break' including routine inspection of implements.

\subsection{Health risks}

The experts were asked to evaluate the risk for developing low back pain posed by exposure to $\mathrm{WBV}$, trunk rotation and if the risks increased if the exposures were combined. The results 
to this question are depicted in Figure 3. The VE and PE considered a risk for LBP from WBV, however the majority of the OE did not perceive this risk to be present. Most experts agreed there exists a risk of LBP from exposure to trunk rotation. More of the vibration experts considered WBV to be a greater risk for LBP than trunk rotation. When considering if the risk for LBP increases if the exposures are combined, 57\% of OE, $78 \%$ of VE and $62 \%$ of PE confirmed that they considered the risks do increase. Additionally, only $6 \%$ of OE and 7\% of VE do not think the risks increase.

\section{[FIGURE 3 ABOUT HERE]}

In order to consider the risk combinations further, PE and VE groups were asked to assess the relative risk (from 'not at all' to 'extremely') of nine predetermined exposure combinations, with trunk rotation and WBV as variables. Table 2 shows the PE and VE group responses, giving the percentage of responses for each rating. In all cases the opinions showed that the assessed risk increased with increasing vibration magnitude and degree of trunk rotation.

\section{[TABLE 2 ABOUT HERE]}

There was no statistically significant differences in the risk assessments between the groups ( $>>0.05$, Chi Squared).

\subsection{Risk Assessment}

When asked to specify time limits for typical exposure combinations involving trunk rotation with and without WBV, all experts were in agreement that exposure duration should be reduced when vibration exposure is combined with trunk rotation, see Table 3 . Although the respondent groups gave different duration responses, the relation between the accepted durations for each condition is the same for each group. In all cases, the acceptable exposure duration halved at least between the low exposure combination (no rotation, no vibration) to 
the high exposure combination (high rotation with vibration), the VE acceptable exposure for the low exposure are 13x that for the high exposure, and 26x the high exposure for the PE.

\section{[TABLE 3 ABOUT HERE]}

The differences between the recommended exposure durations were compared, there were significant differences between the groups for all exposure conditions, $\mathrm{p}<0.01$ (Kruskall Wallis).It can be seen from the analysis that the level of rotation is considered more important than the presence of WBV when experts are asked to rate acceptable exposure durations. The relation between the experts' judged durations is shown in Figure 4. A large proportion of the OE group (45\%) disputed the idea of formalised exposure time limits, adding in the comments box statements including 'until the job is completed' 'if I can do it at my age, anyone can' and 'increase the number of breaks taken'.

\section{[FIGURE 4 ABOUT HERE]}

Those with experience in WBV risk assessment (VE, N=9; PE, N=5) were asked to consider at what level in vibration risk assessment the operators' posture should be considered. All PE (100\%) and the majority of VE (89\%) agreed that there is a need for acknowledgement of additional risk at risk assessment level. A lower proportion (PE, 80\%, VE, 67\%) thought there should be an inclusion within standards, and fewer, although still a majority (PE, 80\%, VE, 56\%) concurred that a consideration of combined exposures should be recognised within legislation. Many felt that further investigation was required to quantify the nature of any interactions. Others pointed to a new ISO standard that is under development. 


\subsection{Body part discomfort}

Experts marked on body outlines where they considered discomfort may develop if an individual was exposed to WBV and trunk rotation. The results were categorised by body segment and are shown in Figure 5. All groups associated discomfort in the lower back with WBV. The OEs associated trunk rotation with neck discomfort, unlike PE and VE groups. When considering combined exposures, all experts judged an increase in discomfort; OE reported discomfort in the right shoulder.

\section{[FIGURE 5 ABOUT HERE]}

OE $(n=32)$ provided details on which tasks caused them particular discomfort. Hedge cutting and ploughing were the most common responses, with 5 and 4 operators respectively noting the discomfort caused by this task. Rotation is clearly a factor with comments from OEs including "any jobs requiring rearward vision” and "all jobs that require almost continuous rotation of the head". However, WBV also appears to be an issue with "hit head on cab roof when going over rough ground”, “driving on road results in neck problems” and "very rough travel affects visibility of the implement”. OEs also commented on cab ergonomics, including problems with insufficient roof height and the placement of controls in the cab. In particular, when operators are required to twist rearwards, controls placed low and forward on the side panel are difficult to reach.

\subsection{Evaluation of previous research}

In consideration of the benefits of previous recommendations made in the literature and guidance, the experts had varied responses. The results are shown in Figure 6. In 
consideration of a full backrest seat, the majority of OE and VE would recommend, however the majority of PE would not. $100 \%$ of the operators questioned would recommend a swivel seat for the exposure considered, along with the majority of VE, but no consensus was seen between the PE responses. 100\% of VE, and the majority of PE would recommend enlarged mirrors in contrast to the majority (61\%) of OE who would not recommend this addition. The same pattern is visible with the CCTV, where both the PE and VE majorities would recommend, but the OE would not. A low proportion of the PE responded to this question, which it is suggested was due to their unfamiliarity with the task example.

\section{[FIGURE 6 ABOUT HERE]}

OE respondents were asked if there were any other ways they perceived the issues described above could be reduced. Cab and seat suspension were mentioned by a large number $(\mathrm{n}=15)$ of OE. Other recommendations included better lever placement, arm support, shorter working periods and task rotation. The other issue mentioned included the placement of implements to the front of the tractor, although it was thought by those suggesting that this would affect visibility and affordability. A contractor, with high working hours and much experience suggested greater degree of seat base rotation (most seats only rotate to $20^{\circ}$ ), suggesting that increasing this to $75-90^{\circ}$ would reduce the discomfort felt when trunk rotation is required for task completion.

\section{Discussion}

This survey utilised a mixed-mode method to collect expert opinion. This involved the use of both paper-based and web-based surveys. There was no clear disparity between the results gained by the paper- or internet-based surveys. Others have previously concluded that the use of either method elicit similar responses (Gosling et al., 2004). The value of survey results is 
often interpreted in part through the non-response rate, which can demonstrate the number of individuals included in the sample not willing or able to complete the survey. The response rate for this survey was impossible to calculate from the online invitation in discussion forums, as the number receiving the invite to participate cannot be determined. If the main aim of the response rate is to determine if the responses are unbiased and typical of the target population, a response (or non-response) rate may not be the best approach (Johnson \& Owens, 2003). The demographics of each of the sample groups indicate a reasonable spread amongst the specialist areas, therefore indicating that typical responses are likely to have been included amongst the data. The numbers of responses are low when considering the total number of professionals working in each of the specific areas. This is an acknowledged weakness of the study. Attempts were made to increase response rates, both by offering a choice of completion method, and through face to face invitations to complete. The number of VE who completed the questionnaire is small in comparison to the other response groups $(\mathrm{VE}=13)$, and this results in a limitation in the interpretation of the responses from this cohort. There are small numbers of experts working in this field in the UK, nevertheless an increased response rate would allow the conclusions from this cohort to be trusted to a greater extent.

In this study three groups with knowledge and expertise in the exposures commonly experienced by agricultural tractor drivers were chosen as representative of the key stakeholders in the area. However, it is conceded that it may have been beneficial to include a response group from a medical and biomechanics backgrounds to allow for completeness. The desire to attain responses and opinions from both operators and academic experts on the same issues meant that the investigation in each subject area had to remain simple enough for others to understand. The benefits being that a clear comparison between response groups is easily available, however, the information gleaned from the experts could have been greater 
if separate questionnaires were developed for each area of expertise.

There were no female responses to the operator questionnaire, however it is known that females do operate the machinery, and their opinion on their specific difficulties with operation may have been particularly insightful.

Some of the responses from the experts suggested that their familiarity with the types of exposures was low. This may have affected their responses, although it is suggested that this would have been of greatest effect in the technical recommendations section, where the responses were more varied among the groups. In the risk assessment and discomfort mapping, there was generally a majority consensus, suggesting agreement among the groups Despite the evidence in the literature that links WBV to LBP in agriculture (e.g. Bovenzi and Betta, 1994; Lings and Leboef, 2000), only 85\% (n=11) of VE questioned considered this risk to be present. Of the PE, only $65 \%$ considered this impact, and in contrast the majority of OE (45\%) did not consider WBV to be a causal factor in the development of LBP. This suggests that the requirements of the $\mathrm{PA}(\mathrm{V}) \mathrm{D}$ for those at risk of vibration-related injury to receive training and education is either not happening, or is not effective. The daily working hours indicated by the OE responses suggest that assessing risks posed by equating risk over a 'normal' 8 hour day underestimate exposure times. The exposures that the expert groups were asked to consider were standardised to facilitate comparison of responses. It is acknowledged that in reality many tasks are composed of periods of high trunk rotation and then periods of low trunk rotation. The use of a task description for the exposures was considered, but it was felt that the risk of differing interpretations of the levels of trunk rotation and/or vibration would invalidate comparison either between or within expert groups. 
When controlling the risks posed by a hazard, reduction at source is often the preferred solution. However, when this is not an available option, reducing or controlling the exposure duration is utilised. For WBV exposure, the PA(V)D provides guidance on reducing the durations an operator may be exposed for increasing magnitudes of WBV. In line with this practice, all of the experts reduced their recommended exposure duration with increasing twist and vibration magnitudes. Although all experts had the reduction with increasing magnitude in common, there was disparity between the operators and academic expert on the durations themselves. Previous studies where the length of acceptable exposure has been investigated showed that increasing posture had a greater effect on reducing the acceptable exposure time than vibration level (Wikstrom, 1994). In this study, the maximum acceptable expsosure time given by the test subjects was 5hours for a $1 \mathrm{~m} / \mathrm{s}^{2}$ vibration condition with twist, which is similar to the times given by the operator experts. Only 55\% of operators provided a duration recommendation, the remainder affirming that despite any associated risk, they would continue with the task until completion. This highlights an issue of risk minimisation in agriculture and emphasises the importance to use engineering solutions for risk reduction strategies first, in an attempt to avoid the issue of non-compliance. Using the data provided in the demographics section regarding exposures that $61 \%$ of operators are operating a tractor for in excess of 10 hours per day, this itself exceeds the maximum durations provided for the 'no twist, no vibration' task by all of the experts.

Body maps are often used to evaluate self-reported and expert opinion of exposures (e.g. Robb and Mansfield, 2007; Kuorinka et al., 1987). In this study, the body map is utilised for comparison between expert's predictions of the localization of discomfort during exposures. This exercise provides an opportunity for comparison of operator first-hand experience of the exposures and academic experts’ experience in judging risk scenarios. In general there was a 
consensus between the 3 response groups on where symptoms would display. The low back area was represented in all but one occasion (trunk rotation, OE). This is in agreement with the multifactorial nature of LBP development (Frymoyer et al., 1983). The prevalence of the right shoulder in the OE responses may be related to control placement in the cab (banked to the right of the operator) and the usual direction of twist (in general to the right). This issue of control placement is noted by others (Donati, 2002). The location of discomfort development under simulated agricultural driving tasks has been previously investigated by others (Donati et al., 1982), where the predominant areas of discomfort were found to be the lumbar back and neck, in static postures, and right thigh, arm and shoulder in dynamic twisting. Wikstrom (1993) explored the nature of the discomfort felt by the operators, the location of the discomfort (to be marked freehand on an outline drawing on the body), and also to rate the acceptable driving time for the exposure they had experienced. The location of discomfort in both the present study and those in the literature is similar. In the OE response group, the thighs were marked as a location for discomfort by $36 \%$ of respondents when assessing the combined exposure scenario; this was not seen in either the PE or VE responses. It has previously been suggested that thigh discomfort in seated driving tasks may be due to poorly fitted (Gyi and Porter, 1999) or improperly adjusted seats (Magnusson and Pope, 1998). Others have reported in the literature that the level of rated discomfort increases when both stressors are experienced together (Wikström 1993; Zimmermann and Cook 1997), which is concurrent with the risk rated by all expert groups here.

There was overwhelming support for the introduction of swivel seats to reduce discomfort from the operator experts (100\%). The posture experts in general did not recommend the use of swivelling seats, and it was suggested by some that the stress of the rotation will shift discomfort from the neck to other body parts, as different parts of the body take the strain in 
the new posture dictated by the rotated seat. Although less consensus was present for the other additions, it was noted amongst respondents that these additions may be beneficial in other task scenarios, so this should not be used to stipulate their exclusion from future tractor design iterations.

The operator group were asked to consider which tasks caused the greatest discomfort. Those requiring twist with a force requirement and high levels of vibration, particularly shock vibration were well represented, such as the use of 'pile drivers' in fence construction. In this instance the operator is required to rotate to the rear to monitor the placement of a ram at the back of the tractor, and when the ram is activated there is a large shock component as the 'pile' (usually in the form of a wooden stake) is driven into the ground. Control placement, armrest length, insufficient head room and requirement for foot pedal force were all issues that operators raised as causing particular discomfort. Many of these issues may conceivably be resolved with some ergonomics intervention at the design phase, as has been proven in other situations (Donati, 2002).

In this study three groups with knowledge and expertise in the exposures commonly experienced by agricultural tractor drivers were chosen as representative of the key stakeholders in the area. The desire to attain responses and opinions from both operators and academic experts on the same issues meant that the investigation in each subject area had to remain simple enough for others to understand. However, this allows for a clear comparison between response groups to be made. In future, single-discipline expert groups could be targeted to allow for more detailed questionnaires to be used. It is suggested that this multidisciplinary approach to investigating a topic may be the optimal approach in future studies of this form. 


\section{Conclusions}

Results show the consensus that exposing operators to WBV and trunk rotation simultaneously increases perceptions of risk for low back pain. Operators reported discomfort in the right shoulder and thighs when exposed to both risk factors. This was not highlighted in the academic responses. Those with experience in risk assessment were in agreement that the issue of postural stress should be considered when assessing the risk from vibration exposure. Interventions aimed at reducing the requirement for twist and improving seat discomfort should therefore be prioritised as a risk reduction strategy for off-road drivers. A large proportion of the operator respondents were operating a tractor for in excess of 10 hours per day, therefore it is unlikely that recommendations for risk reduction based on exposure limitation would be particularly effective.

\section{References}

Bottoms DJ, Barber TS. 1978, A swivelling seat to improve tractor drivers’ posture. Applied Ergonomics, 9,2, 77-84.

Bovenzi M, Betta A. 1994, Low-back disorders in agricultural tractor drivers exposed to whole-body vibration and postural stress, Applied Ergonomics, 25, 231-241

Bovenzi M. 2009, Metrics of whole-body vibration and exposure-response relationship for low back pain in professional drivers: a prospective cohort study, International Archives of Occupational and Environmental Health, 82, 7, 893-917.

Geoffrey David, Valerie Woods, Guangyan Li, Peter Buckle, The development of the Quick Exposure Check (QEC) for assessing exposure to risk factors for work-related musculoskeletal disorders, Applied Ergonomics, Volume 39, Issue 1, January 2008, Pages 57-69

DEFRA, Department for Food and Rural Affairs, 2008. Agriculture statistics in your pocket, http://statistics.defra.gov.uk/esg/publications/auk/default.asp. Date accessed: 30/03/08 
Evaluation of Working Postures and Movements in relation to Machinery: EN 1005-4, 2005

Donati, P., Boldero, A. G., \& Stayner, R. M. 1982. Simulation of the tractor driver's task for seat comfort assessment. Natn Inst Agric Engng, Silsoe, Divisional Note DN/1108 (unpublished).

Donati P, 2002, Survey of technical preventative measures to reduce whole-body vibration effects when designing mobile machinery. Journal of Sound and Vibration, 253, 1, 169183

European Parliament and the Council of the European Union (2002) Directive 2002/44/EC on the minimum health and safety requirements regarding the exposure of workers to the risks arising from physical agents THE EUROPEAN VIBRATION DIRECTIVE 479 (vibration). Official Journal of the European Communities, OJ L177, 6.7.2002, 13-9. Frymoyer JW, Pope MH, Clements JH, Wilder DG, MacPherson B and Ashikaga T, 1983, Risk factors in low-back pain. An epidemiological survey, Journal of Bone \& Joint Surgery., 65, 213-218.

Frymoyer JW, Pope MH, Costanza MC, Rosen JC, Goggin JE and Wilder DG. 1998, Epidemiologic studies of low back pain, Spine, 215, 4, 965-976.

Gosling, S. D., Vazire, S., Srivastava, S., \& John, O. P. (2004). Should we trust web-based studies? A comparative analysis of six preconceptions about internet questionnaires. American Psychologist, 59(2), 93.

Gyi, D.E., Porter, J.M., 1999. Interface pressure and the prediction of car seat discomfort. Applied Ergonomics 30, 99-107.

Hignett S, McAtamney L, 2000. Rapid Entire Body Assessment (REBA), Applied Ergonomics, 31, 201-205 
Hoy D, March L, Brooks P, Woolf A, Blyth F, Vos T, Buchbinder R, 2010. Measuring the global burden of low back pain, Best practice research Clinical rheumatology, 24, 2, 155 165

HSE statistics, 3 year average back pain prevalence 2005/6 - 2007-8 http://www.hse.gov.uk/statistics/lfs/0708/backind2_3yr.htm, date accessed 30/11/09

Health and Safety Executive, 2005. The Control of Vibration at Work Regulations. www.hse.gov.uk

Institute of Ergonomics and Human Factors, IEHF, Knowledge areas, http://www.ergonomics.org.uk/knowledge-areas/ date accessed 30/11/09

International Organisation for Standardisation. Mechanical vibration and shock - evaluation of human response to whole body vibration - part 1: general requirements, ISO 26311:1997, 1997

Johnson, T., \& Owens, L. 2003. Survey response rate reporting in the professional literature. In 58th Annual Meeting of the American Association for Public Opinion Research, Nashville.

Kuorinka I, Jonsson B, Kilbom A, Vinterberg H, Biering-Sørensen F, Andersson G and Jørgensen K, 1987, Standardised Nordic questionnaires for the analysis of musculoskeletal symptoms, Applied Ergonomics,18, 3, 233-237

Lings S, Leboeuf-Yde C, 2000, Whole-body vibration and low back pain: a systematic, critical review of the epidemiological literature 1992-1999. International Archives of Occupational and Environmental Health 73,5, 290-297.

Magnusson ML and Pope MH. 1998, A review of the biomechanics and epidemiology of working postures (It isn’t always vibration which is to blame), Journal of Sound and Vibration 215,4 965-976 
McAtamney, L., \& Nigel Corlett, E. 1993. RULA: a survey method for the investigation of work-related upper limb disorders. Applied ergonomics, 24(2), 91-99

Mehta CR, Tiwari VK. 2000, Seating discomfort for tractor operators: a critical review, International Journal of Industrial Ergonomics, 25, 661-674

Okuniribido OO, Magnusson M, and Pope MH., 2008, The role of whole body vibration, posture and manual materials handling as risk factors for low back pain in occupational drivers, Ergonomics, 51, 3, 308-329

Pheasant and Haslegrave, 2006, Bodyspace: Antropometry, ergonomics, and the design of work, $3^{\text {rd }}$ Ed, CRC Press, London

Punnett L, Pruss-Utun A, Nelson DI, Fingerhut MA, Leigh J, Tak S, Phillips S, 2005. Estimating the global burden of low back pain attributable to combined occupational exposures, American Journal of Industrial Medicine, 48, 6, 459-469

Robb MJ, Mansfield NJ 2007, Self-reported musculoskeletal problems amongst professional truck drivers, Ergonomics, 50,6, 814-827

Sandover, J. 1998. High acceleration events: An introduction and review of expert opinion. Journal of Sound and Vibration, 215(4), 927-945.

Schwarze S, Notbohm G, Dupuis H, Hartung E, 1998. Dose-response relationships between whole-body vibration and lumbar disk disease - a field study on 388 drivers of different vehicles, Journal of Sound and Vibration 215, 4, 613-628

Sjøflot L. 1980, Means of improving a tractor drivers working posture, Ergonomics, 23, 751761

Sorainen, E., Penttinen, J., Kallio, M., Rytkönen, E., \& Taattola, K. 1998. Whole-body vibration of tractor drivers during harrowing. American Industrial Hygiene Association, 59(9), 642-644

Survey Monkey, 2009. www.surveymonkey.com/ 
Tiemessen IJH, Hulshof CTJ, Frings-Dresen MHW. 2008, Low back pain in drivers exposed to whole body vibration: analysis of a dose-response pattern, Occupational and Environmental Medicine, 65, 667-675

Torén, A., \& Öberg, K. 2001. IT_-Information Technology Change in Twisted Trunk Postures by the Use of Saddle Seats—a Conceptual Study. Journal of agricultural engineering research, 78(1), 25-34

Wikstrom 1993 Bengt-O. Wikström, Effects from twisted postures and whole-body vibration during driving, International Journal of Industrial Ergonomics, Volume 12, Issues 1-2, August 1993, Pages 61-75, ISSN 0169-8141, http://dx.doi.org/10.1016/0169-8141(93)90038F.

Wikstrom BO, Kjellberg A, Landstrom U. 1994, Health Effects of long-term occupational exposure to whole-body vibration: A review. International Journal of Industrial Ergonomics 14, 273-292

Wilder DG, Pope MH. 1996, Epidemiological and aetiological aspects of low back pain in vibration environments: an up-date. Clinical Biomechanics, 11, 61-73 
Figure 1 Body outline for marking of discomfort.

Figure 2 Length of experience for the three response groups OE, PE and VE.

Figure 3 The risk of developing low back pain from WBV, trunk rotation, and consideration if the risk increases from combined exposures (OE $n=33$; $P E n=37 ; V E n=13$ ).

Figure 4 The mean acceptable working durations for the specified exposures

Figure 5 Shaded areas indicate where $>40 \%$ of experts predicted discomfort under the different exposure combinations (PE, n=35; VE, $n=13$; OE, $n=22$ )

Figure 6 Consideration by experts of previous recommendations from the literature for reducing risk for LBP from occupational exposures with trunk rotation and WBV exposure. (OE, n=23; VE, n=13; PE, n=9).

Table 1 Methods of recruitment for the target respondent groups.

Table 2 Level of risk associated with whole-body vibration and trunk rotation exposure combinations, expressed as percentage of expert responses (PE $n=38$; VE $n=13$ ).

Table 3 Suggested exposure time limit for exposures across the three expert groups, for varying exposure combinations of trunk twist and WBV exposure (mean, with range specified in parenthesis) 
Table 3 Methods of recruitment for the target respondent groups.

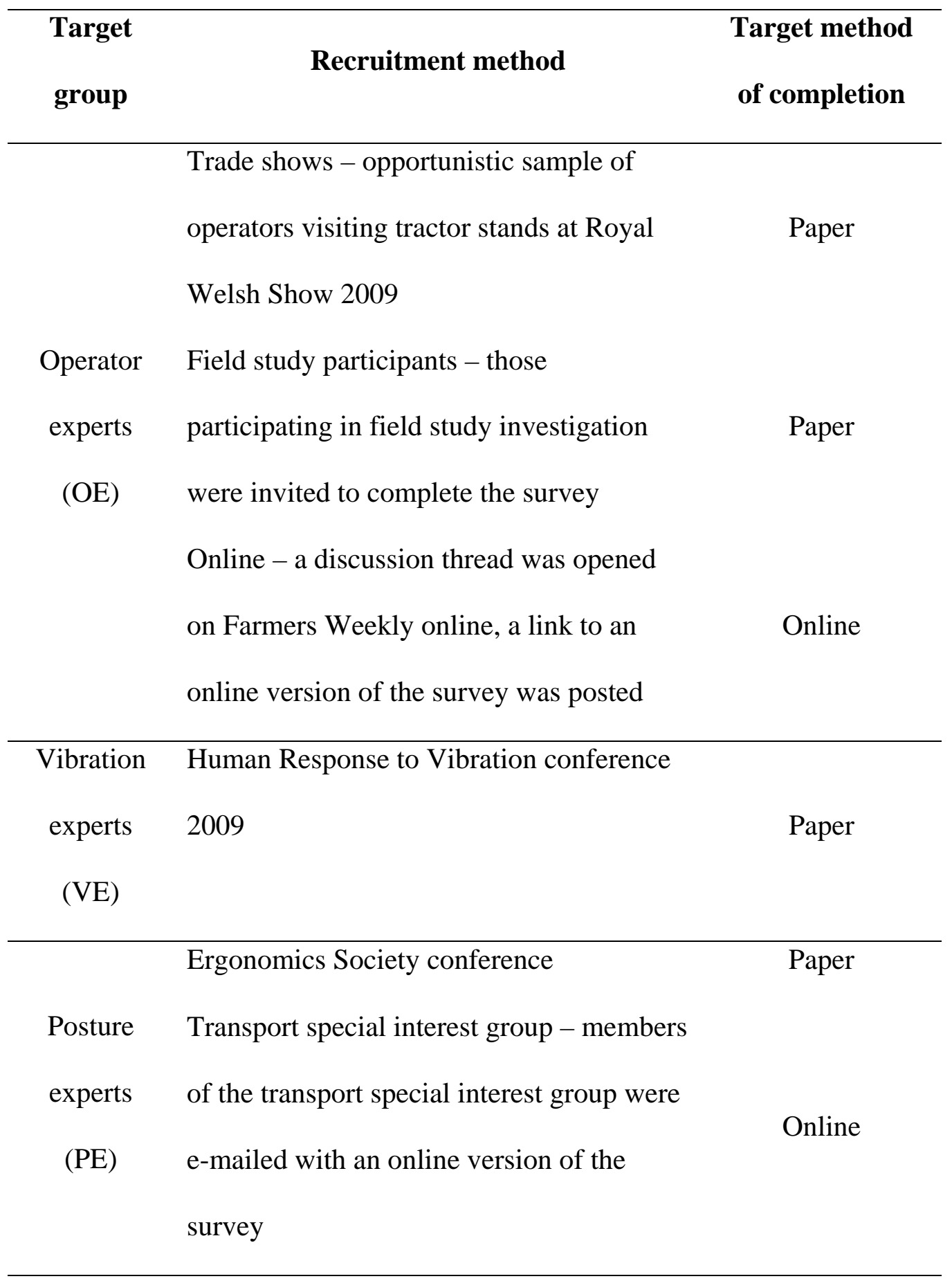




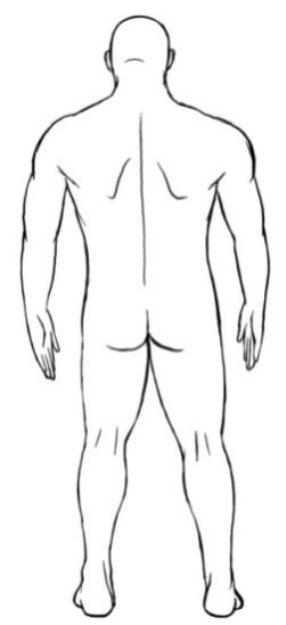

Figure 1 Body outline for marking of discomfort.

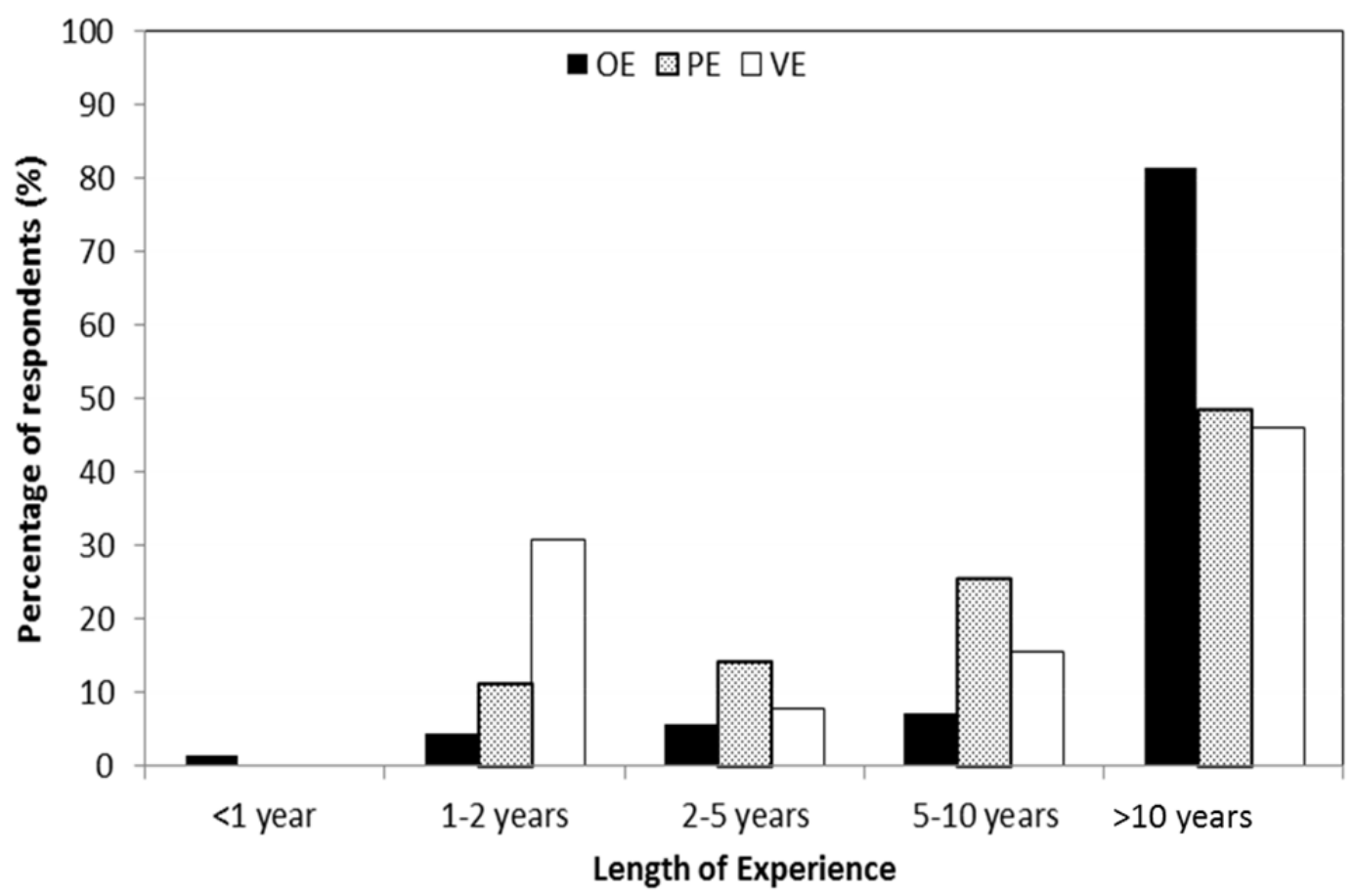

Figure 2 Length of experience for the three response groups OE, PE and VE. 


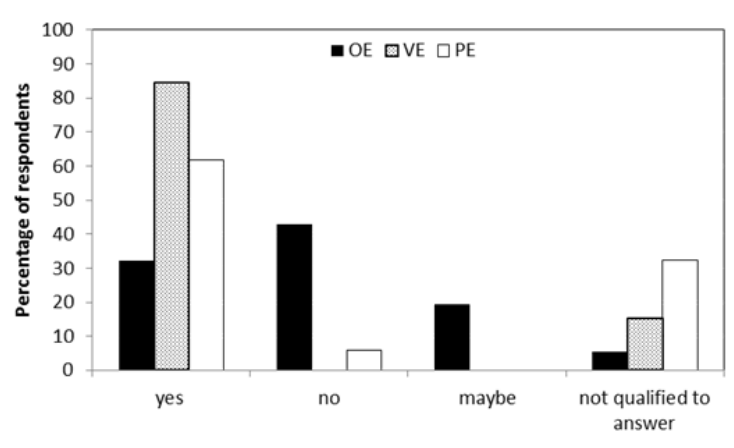

WBV exposure only

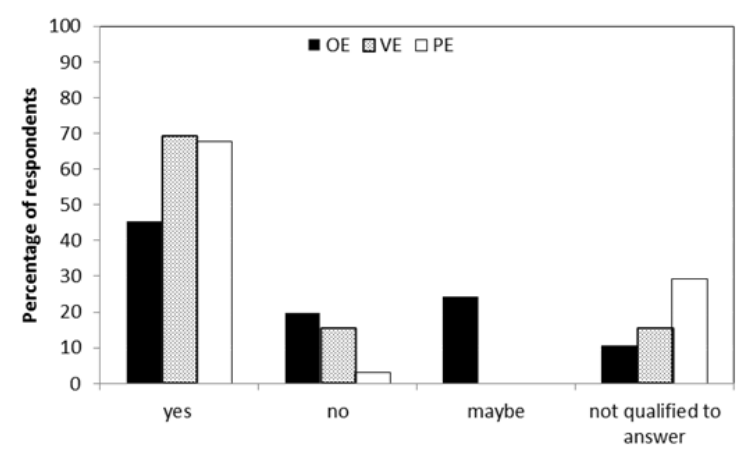

trunk rotation only

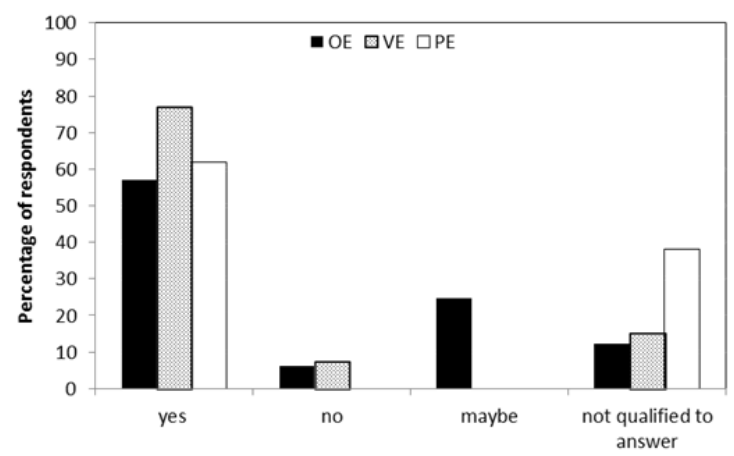

WBV and trunk rotation

Figure 3 The risk of developing low back pain from WBV, trunk rotation, and consideration if the risk increases from combined exposures (OE $n=33$; $P E n=37$; VE $n=13$ ). 
Table 4 Level of risk associated with whole-body vibration and trunk rotation exposure combinations, expressed as percentage of expert responses (PE n=38; VE n=13).

\begin{tabular}{|c|c|cc|cc|cc|cc|cc|}
\hline \multicolumn{2}{|c|}{ Combination } & \multicolumn{2}{|c|}{ Not at all } & \multicolumn{2}{|c|}{ Slightly } & \multicolumn{2}{|c|}{ Moderately } & \multicolumn{2}{|c|}{ Very } & \multicolumn{2}{|c|}{ Extremely } \\
Twist & Vibration & PE & VE & PE & VE & PE & VE & PE & VE & PE & VE \\
\hline $0^{\circ}$ & Low & $43 \%$ & $67 \%$ & $57 \%$ & $33 \%$ & $0 \%$ & $0 \%$ & $0 \%$ & $0 \%$ & $0 \%$ & $0 \%$ \\
$0^{\circ}$ & Medium & $5 \%$ & $22 \%$ & $62 \%$ & $44 \%$ & $29 \%$ & $33 \%$ & $5 \%$ & $0 \%$ & $0 \%$ & $0 \%$ \\
$0^{\circ}$ & High & $0 \%$ & $0 \%$ & $23 \%$ & $11 \%$ & $50 \%$ & $44 \%$ & $18 \%$ & $44 \%$ & $9 \%$ & $0 \%$ \\
$70^{\circ}$ & Low & $0 \%$ & $22 \%$ & $50 \%$ & $56 \%$ & $45 \%$ & $22 \%$ & $0 \%$ & $0 \%$ & $5 \%$ & $0 \%$ \\
$70^{\circ}$ & Medium & $0 \%$ & $11 \%$ & $5 \%$ & $22 \%$ & $65 \%$ & $67 \%$ & $25 \%$ & $0 \%$ & $5 \%$ & $0 \%$ \\
$70^{\circ}$ & High & $0 \%$ & $0 \%$ & $0 \%$ & $11 \%$ & $26 \%$ & $22 \%$ & $53 \%$ & $44 \%$ & $21 \%$ & $22 \%$ \\
$170^{\circ}$ & Low & $0 \%$ & $11 \%$ & $0 \%$ & $11 \%$ & $30 \%$ & $56 \%$ & $60 \%$ & $22 \%$ & $10 \%$ & $0 \%$ \\
$170^{\circ}$ & Medium & $0 \%$ & $0 \%$ & $0 \%$ & $0 \%$ & $5 \%$ & $33 \%$ & $60 \%$ & $56 \%$ & $35 \%$ & $11 \%$ \\
$170^{\circ}$ & High & $0 \%$ & $0 \%$ & $0 \%$ & $0 \%$ & $0 \%$ & $22 \%$ & $16 \%$ & $11 \%$ & $84 \%$ & $67 \%$ \\
\hline
\end{tabular}


Table 3 Suggested exposure time limit for exposures across the three expert groups, for varying exposure combinations of trunk twist and WBV exposure (mean, with range specified in parenthesis)

\begin{tabular}{ccccccc}
\hline & No twist, & Med. twist, & High twist & No twist, & Med. twist, & High twist, \\
& no WBV & no WBV. & no WBV. & with WBV. & with WBV. & with WBV. \\
\hline VE & $06: 30$ & $01: 45$ & $00: 53$ & $03: 00$ & $01: 00$ & $00: 30$ \\
$(\mathrm{~N}=13)$ & $(02: 00$, & $(00: 30$, & $(00: 20$, & $(01: 30$, & $(00: 50$, & $(00: 25$, \\
& $08: 00)$ & $02: 00)$ & $01: 00)$ & $03.45)$ & $01: 00)$ & $00.30)$ \\
\hline $\mathrm{PE}$ & $04: 00$ & $01: 00$ & $00: 15$ & $02: 00$ & $00: 30$ & $00: 10$ \\
$(\mathrm{~N}=20)$ & $(02: 00$, & $(00: 30$, & $(00: 10$, & $(02: 00$, & $(00: 20$, & $(00.00$, \\
& $08: 00)$ & $01: 20)$ & $00.30)$ & $03: 00)$ & $01: 10)$ & $00: 25)$ \\
\hline $\mathrm{OE}$ & $09: 00$ & $08: 00$ & $04: 00$ & $06: 00$ & $05: 00$ & $03: 30$ \\
$(\mathrm{~N}=26)$ & $(08: 00$, & $(05: 30$, & $(01: 00$, & $(04: 30$, & $(04: 00$, & $(02: 00$, \\
& $12: 00)$ & $09.30)$ & $08: 00)$ & $10.00)$ & $06.50)$ & $04: 00)$ \\
\hline
\end{tabular}




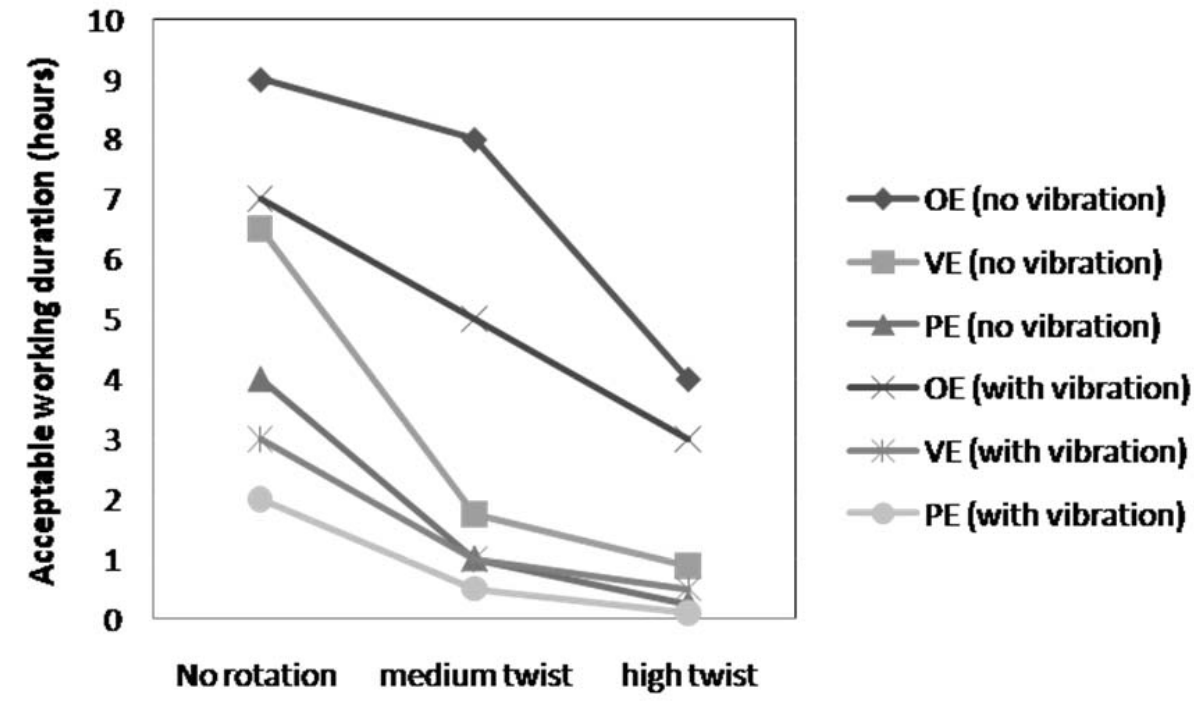

Figure 4 The mean acceptable working durations for the specified exposures 


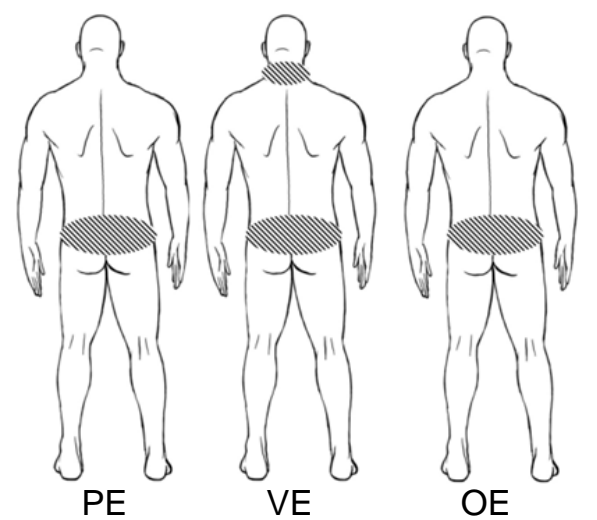

Whole-body vibration

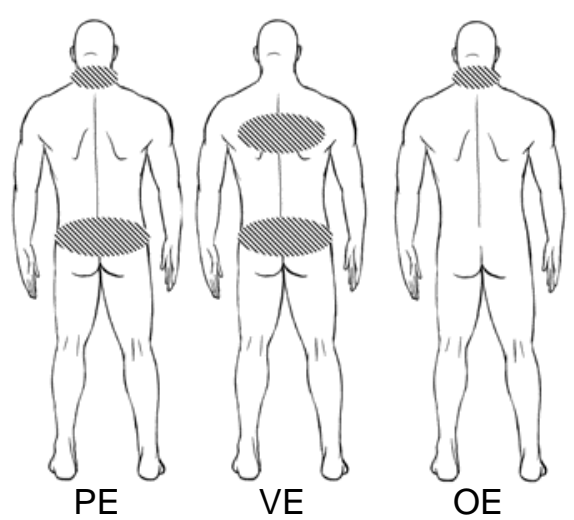

Trunk rotation

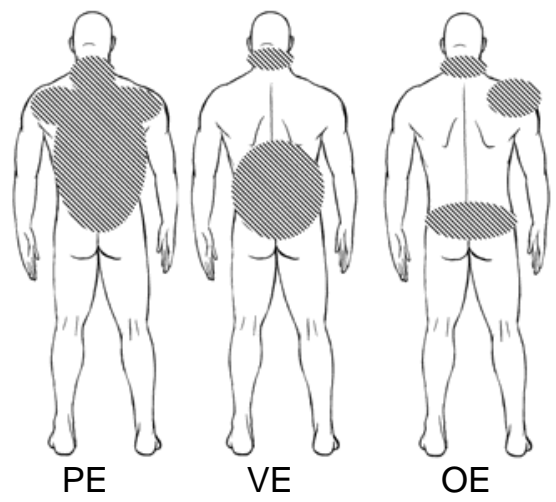

Whole-body vibration and trunk rotation

Figure 5 Shaded areas indicate where $>40 \%$ of experts predicted discomfort under the different exposure combinations (PE, $n=35$; VE, $n=13$; OE, $n=22$ ) 


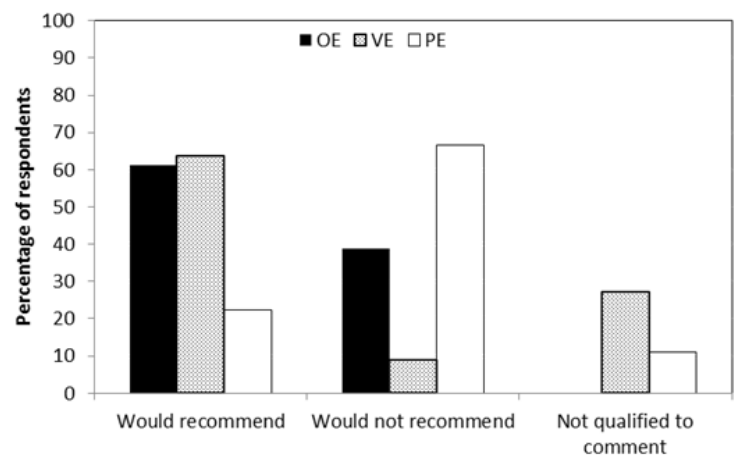

Full backrest seat

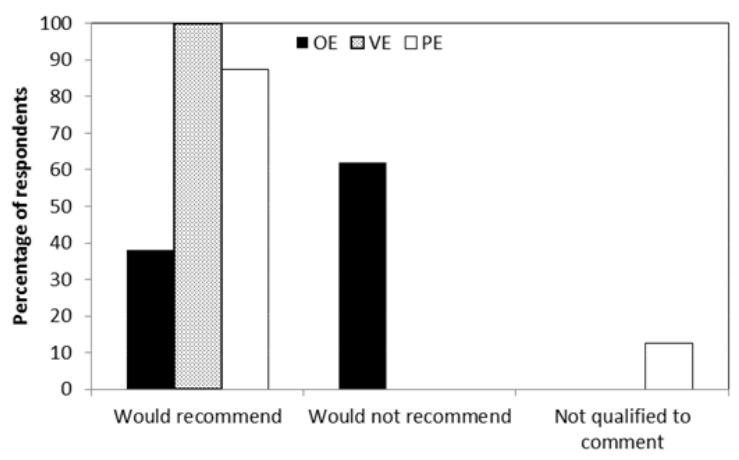

Enlarged mirrors

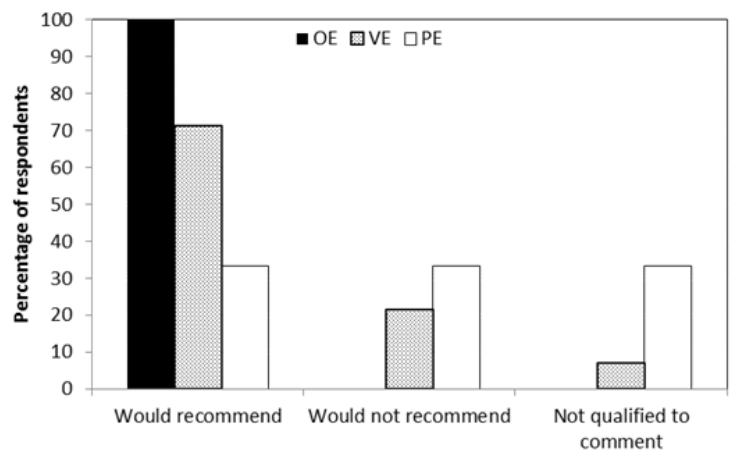

Swivelling seat

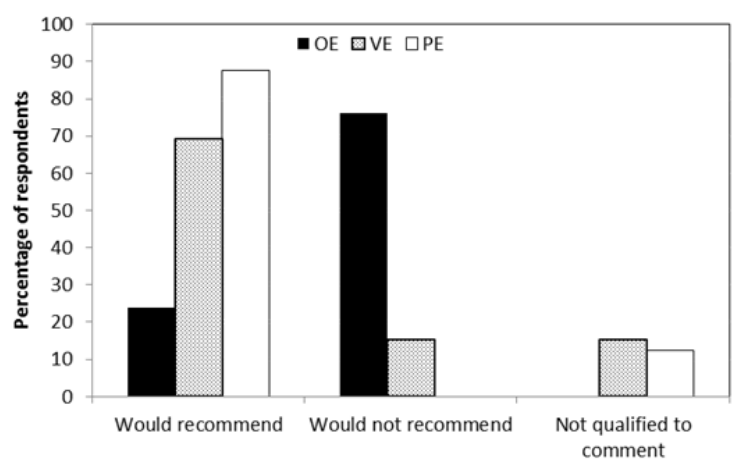

CCTV

Figure 6 Consideration by experts of previous recommendations from the literature for reducing risk for LBP from occupational exposures with trunk rotation and WBV exposure. (OE, n=23; VE, n=13; PE, n=9). 